\title{
PULSATILE OCULAR BLOOD FLOW IN EYES WITH UNTREATED CHOROIDAL MELANOMA
}

\author{
Y. C. YANG, D. KENT, C. H. FENERTY, A. S. KOSMIN and B. E. DAMATO \\ Liverpool
}

\section{SUMMARY}

Purpose: To investigate the effect of choroidal melanoma on pulsatile ocular blood flow (POBF).

Methods: Seventeen patients (10 men and 7 women) with unilateral untreated choroidal melanoma and 22 controls matched for age and sex were studied. Intraocular pressure (IOP), pulse amplitude (PA) and POBF were measured using the OBF Tonograph. In each patient, mean inter-ocular differences were analysed using the paired $t$-test. The correlation coefficient between tumour thickness and POBF was calculated. To assess the variation of this parameter, the coefficient of variation for three repeated readings was determined for healthy and affected eyes.

Results: In the control group, there was no significant difference between eyes in any parameter. In patients with melanoma, there was no significant difference in IOP and PA between affected and unaffected eyes. Mean POBF was significantly higher in affected eyes $\left(1040 \mu \mathrm{l} \mathrm{min}^{-1}\right)$ than unaffected eyes $\left(876 \mu \mathrm{l}\right.$ min $\left.^{-1}\right)$ $(p=0.003)$. There was no correlation between tumour thickness and absolute POBF $(r=-0.24)$ or between tumour thickness and inter-ocular difference in POBF between affected and unaffected eyes $(r=-0.17)$. Mean coefficient of variation of three repeated readings of POBF was $7.76 \%$ in healthy eyes and $8.97 \%$ in affected eyes.

Conclusions: These findings suggest a high tumour blood flow or a global increase in choroidal blood flow in the presence of melanoma. POBF measurement may be useful in the clinical assessment of eyes with choroidal melanoma.

Measurements of tumour circulation may allow prediction of tumour responsiveness to chemotherapy or immunotherapy and may also be of prognostic value with respect to metastatic disease. ${ }^{1,2}$ Histolo-

Correspondence to: Y. C. Yang, St Paul's Eye Unit, Royal Liverpool University Hospital, Prescot Street, Liverpool L7 8XP, UK. Tel: +44 (151) 706 2000. Fax: +44 (151) 7065861 . gical studies have shown a correlation between tumour vascularity and metastatic disease.,

Although qualitative data can be obtained on the tumour vascularity with techniques such as A- and Bmode ultrasonography, fluorescein angiography and indocyanine green angiography, ${ }^{5-8}$ an accurate and non-invasive method of measuring circulation in a uveal melanoma may provide a valuable means of assessing tumour activity. Duplex ultrasound with colour Doppler imaging (CDI) has been used in several studies to quantify blood flow in choroidal melanoma. ${ }^{9,10}$ In this method, the magnitude of pulsatile flow is expressed in terms of frequency shifts rather than velocity, as the angle between the ultrasound beam and the blood vessels imaged cannot be determined accurately. ${ }^{11}$ As measurements are taken from a small sample area it is uncertain whether such measurements are representative and whether repeated measurements over time from different areas of a tumour can be reliably compared. ${ }^{11,12}$

Pulsatile ocular blood flow (POBF) estimation by tonography using the OBF system was first developed by Langham et al. ${ }^{13,14}$ With this method, the ocular pulse is recorded and analysed mathematically

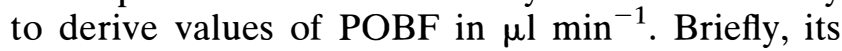
principle is based on converting the pressure curve recorded to a volume curve from known values of pressure/volume relationships of the human globe. The slope of the volume curve, obtained by mathematical differentiation, describes the net flow rate with time. The POBF is then determined from the net flow curve. ${ }^{15-17}$

The aim of this study was to determine whether uveal melanomas cause a measurable change in POBF.

\section{METHOD}

Seventeen patients (10 men and 7 women) with newly diagnosed unilateral choroidal melanoma and 22 age-matched controls ( 13 men and 9 women) were 
selected. All patients gave informed consent to participate in the study, which was approved by the Royal Liverpool University Hospital's Ethics Committee. All patients and control subjects received a full ophthalmic and systemic assessment. Patients with other ocular pathologies, and a history of diabetes or cardiovascular disease, were excluded.

Tumour thickness was measured with a B scan and axial eye length with an A scan. As POBF values can vary with axial length, ${ }^{18}$ control subjects with asymmetrical $(>3 \mathrm{~mm})$ axial lengths were also excluded. POBF was measured using the OBF Tonograph (ver10.0, OBF Labs UK, Cleverton, Wiltshire, UK), which is based on the original Langham OBF system. It consists of a floating tip pneumotonometer, which ensures a constant applanating force for recording the ocular pulse, and a tonograph unit linked to a computer for automatic selection and analysis of consecutive pulses to derive measurements of the various ocular pulse characteristics, including pulse amplitude (PA) and POBF. POBF determined in this way reflects only the pulsatile component of blood flow to all the intraocular structures and does not specifically distinguish the component distributed to the tumour. However, it is non-invasive, easy to measure and is also free from observer error as pulse selection and analysis are computerised and based on consistent criteria.

Following instillation of oxybuprocaine $0.4 \%$ and fluorescein drops, intraocular pressures were measured using Goldmann applanation tonometry before commencement of tonography. Tonographic measurements were taken with the patient rested and seated at a slit lamp using the same OBF Tonograph throughout by a single examiner (Y.C.Y.), who was masked to the laterality of the affected eye. Tonography was repeated three times in each eye, with the first eye chosen at random and alternating between eyes in quick succession to eliminate the effects of first exposure and any variation in ocular blood flow caused by physiological cardiovascular changes. ${ }^{19,20}$ To counter any effects of diurnal variation, ${ }^{21}$ all tonographic measurements were taken in mid-afternoon.
Mean values of $\mathrm{PA}$ in $\mathrm{mmHg}$ and $\mathrm{POBF}$ in $\mu 1 \mathrm{~min}^{-1}$ were calculated for each subject. The correlation coefficients of tumour thickness with both absolute mean POBF and the difference in mean POBF were calculated. To assess the repeatability of POBF measurements, the coefficients of variation $(\mathrm{CV})$ of three successive measurements in affected and unaffected eyes were calculated. The paired $t$-test was used for statistical hypothesis testing.

\section{RESULTS}

The mean age of patients with choroidal melanoma was 55 years (SD 10.9 years). The tumour affected the right eye in 6 patients and the left eye in 11. All tumours were melanotic and had not broken through Bruch's membrane and the retinal pigment epithelium. The tumour thicknesses ranged from $1.92 \mathrm{~mm}$ to $8.51 \mathrm{~mm}$ (mean $4.7 \mathrm{~mm}$ ).

In the control group there was no significant interocular difference in any of the parameters. Patients with choroidal melanoma had a significantly higher POBF in affected eyes $(p=0.003)$ but no difference in IOP or PA between eyes (Table I).

Mean coefficient of variation of three readings was $7.76 \%$ in unaffected eyes and $8.97 \%$ in affected eyes. This difference was not statistically significant $(p=0.33)$. No correlation was found between tumour thickness and absolute POBF $(r=-0.24)$ or between tumour thickness and inter-ocular difference in POBF between affected and unaffected eyes $(r=-0.17)$.

\section{DISCUSSION}

In the healthy eye, over $90 \%$ of ocular blood flow is distributed to the choroid. ${ }^{22,23}$ The physiological ocular pulse which results from the pulsatile nature of blood flow in intra-ocular vessels has been shown to arise from the choroidal vessels. ${ }^{24}$ POBF values which are derived from recordings of the ocular pulse therefore reflect the pulsatile component of blood flow in the choroid. ${ }^{13}$

In this study, we succeeded in our aim of determining whether choroidal melanomas cause a

Table I. Summary of results for the melanoma group and the control group

\begin{tabular}{lccc}
\hline Parameter & Affected eye/right eye ${ }^{\mathrm{a}}(\mathrm{SD})$ & Unaffected eye/left eye $^{\mathrm{a}}(\mathrm{SD})$ & $p$ value \\
\hline Melanoma group $(n=17)$ & $15.05(2.29)$ & $15.89(2.11)$ & 0.12 \\
Mean IOP $(\mathrm{mmHg})$ & $2.48(0.76)$ & $2.33(0.74)$ & 0.21 \\
Mean PA (mmH) & $1040.81(366.58)$ & $876.37(296.71)$ & 0.003 \\
Mean POBF $\left(\mu \mathrm{l} \mathrm{min}{ }^{-1}\right)$ & 22.13 & 22.28 & 0.31 \\
Mean axial length $(\mathrm{mm})$ & & & \\
Control group $(n=22)$ & 15.2 & 15.5 & 0.72 \\
Mean IOP $(\mathrm{mmHg})$ & $2.63(1.00)$ & $2.70(0.70)$ & 0.60 \\
Mean PA $(\mathrm{mmHg})$ & $816.73(238.98)$ & $825.45(181.06)$ & 0.81 \\
Mean POBF $\left(\mu \mathrm{l} \mathrm{min}{ }^{-1}\right)$ & & \\
\hline
\end{tabular}

IOP, intraocular pressure; PA, pulse amplitude; POBF, pulsatile ocular blood flow.

${ }^{a}$ In the melanoma group the affected eye was compared with the unaffected eye; in the control group the right eye was compared with the left eye. 
measurable change in POBF. The higher POBF values observed in eyes with choroidal melanoma indicate that the pulsatile component of choroidal blood flow was increased. Assuming that the blood flow in the healthy choroid remained unaltered, this finding implies that the increase in choroidal blood flow is due to the tumour circulation. It is well established from theoretical analysis and histological morphometric studies on malignant melanomas that an increase in vascularity within a tumour is associated with increased vascular space, blood volume and flow. ${ }^{1}$ In cutaneous melanoma, clinical measurements of blood flow using colour Doppler imaging have been found to correspond to vascular morphology on histological studies. ${ }^{25}$ In uveal melanoma using colour Doppler imaging, Lieb et al. ${ }^{9}$ and Wolff-Kormann et al. ${ }^{10}$ both found increased blood flow parameters in untreated tumours and reduced blood flow in irradiated tumours. In one patient with a treated tumour, Wolff-Kormann reported a recurrence of high blood flow parameters coinciding with tumour recurrence detectable clinically. These findings and those of our study further support a role for ocular blood flow measurement in the assessment of choroidal melanoma.

Although follow-up measurements were not performed in this study we were able to calculate the coefficient of variation of POBF readings repeated in quick succession as an index of reproducibility. The mean coefficient of variation of three repeated readings was below $9 \%(2.3-18 \%)$, which is clinically acceptable. Bosem et l. $^{26}$ also reported a low coefficient of variation of repeated POBF readings (CV $7.5 \%)$ in eyes with glaucoma. There was, however, a wide inter-individual variation in the POBF in eyes with melanomas (SD 366.58), such that there was a considerable overlap of POBF values between the affected eyes and controls. This is due to the inherent inter-individual variability of this parameter, which is also present in healthy eyes. ${ }^{27}$ This emphasises the importance of demonstrating an inter-ocular difference in POBF or changes in POBF over time within an individual's eye when comparing POBF values. Although the mean POBF value in eyes with melanoma is higher than that found in other studies in eyes with other conditions, ${ }^{28-31}$ a direct comparison of POBF measurements made in small groups of patients with different diseases should be avoided due to the high interindividual variation of this parameter.

In this study, no correlation was found between tumour thickness and the absolute POBF or the magnitude of the POBF rise in affected eyes. As the whole tumour circulation contributes to the POBF, it would be more appropriate to correlate tumour volume with POBF. However, due to the irregularity of the tumour shapes, their volumes could not be accurately determined from our recordings of maximal basal dimensions and thicknesses alone. WolffKormann et $a l^{10}$ also reported no correlation between tumour blood flow and thickness with colour Doppler imaging, but in contrast Srivastava et $a l^{32}$ found unrecordable blood flow in thin cutaneous melanomas but higher blood flow in thicker tumours and postulated a relationship between tumour thickness and blood flow.

As the ocular pulse is a pressure pulse due to an afferent bolus of blood to the eye, the pulse amplitude is greater when the globe is less distensible or when the perfusion pressure is increased. The latter is apparent following carotid endarterectomy, which causes an increase in pulse amplitude and POBF by an increase in the perfusion pressure. ${ }^{33}$ Although carotid studies were not performed, the inter-ocular difference in POBF found in the melanoma patients is unlikely to be due to carotid disease as none of the patients had a history of cardiovascular disease or symptoms and signs of carotid occlusion. When ocular rigidity is reduced and the globe is more distensible, the pulse amplitude is reduced as the pressure pulse is converted to a volume pulse. This is seen in high myopia, corneal thinning, staphyloma ${ }^{34}$ and after trabeculectomy. ${ }^{35}$ In such situations when the ocular rigidity has been significantly altered by a pathological process or surgical procedure, POBF measurements are actually invalid as the volume/pressure relationship values for normal globes used in the derivation of POBF are no longer applicable. ${ }^{36}$ The absence of a significant inter-ocular difference in pulse amplitude between affected and unaffected eyes suggests that the ocular rigidity in these patients was not affected by the tumour.

To the best of our knowledge, this is the first study to investigate $\mathrm{POBF}$ in choroidal melanoma. Although POBF has been found to be reduced in certain conditions including glaucoma, cataract and diabetic retinopathy, ${ }^{28-31}$ the significance of such findings is uncertain. Fundamental studies to validate the theoretically derived POBF values and clinical studies to determine reference ranges and variation in health and diseases are required before any meaningful interpretation of this parameter can be made.

In conclusion, this study provides further evidence that tumour blood flow can be quantified indirectly by a non-invasive technique. As it is not possible to verify these techniques with direct, invasive methods, such data must, at present, be interpreted with caution. It is essential at this stage to evaluate not only the applicability of new techniques but also the validity and repeatability of their measurements. With regard to these considerations the measure- 
ment of global pulsatile blood flow changes using the OBF Tonograph may prove to be of value in the assessment of eyes with choroidal melanoma.

Key words: Ocular, Blood flow, Pulsatile, Choroidal, Melanoma.

\section{REFERENCES}

1. Jain RK. Determinants of tumour blood flow: a review. Cancer Res 1988;48:2641-58.

2. Folkman J, Klaysbrun M. Angiogenic factors. Science 1987;235:442-7.

3. Folberg R, Jacob P, Gruman LM, Woolson RF, Jeng $\mathrm{G}$, et al. The morphologic characteristics of tumour blood vessels as a marker of tumour progression in primary human uveal melanoma: a matched casecontrol study. Hum Pathol 1992;23:1298-305.

4. Miller MV, Herdson PB, Hitchcock GC. Malignant melanoma of the uveal tract: a review of the Auckland experience. Pathology 1985;17:281-4.

5. Feleppa EJ, Lizzi FL, Coleman DJ, Yarenko MM. Diagnostic spectrum analysis in ophthalmology: a physical perspective. Ultrasound Med Biol 1986; 12:623-31.

6. Hayreh SS. Choroidal melanomata: fluorescein angiographic and histopathological study. Br J Ophthalmol 1970;54:145-60.

7. McMahon RT, Tso MOM, McLean IW. Histologic correlation of fluorescein angiography in choroidal malignant melanoma. Am J Ophthalmol 1977;83: 836-46.

8. Shields CL, Shields JA, De Potter P. Patterns of indocyanine green videoangiography of choroidal tumours. Br J Ophthalmol 1995;79:237-45.

9. Lieb WE, Shields JA, Cohen SM, Merton DA, Mitchell $\mathrm{DG}$, et al. Color Doppler imaging in the management of intra-ocular tumors. Ophthalmology 1990;97: $1660-4$.

10. Wolff-Kormann PG, Kormann BA, Reidel KG, Hasenfratz GC, Stefani FH, et al. Quantitative colour Doppler imaging in untreated and irradiated choroidal melanoma. Invest Ophthalmol Vis Sci 1992;33:1928-33.

11. Taylor KJW, Holland S. Doppler US. I. Basic principles, instrumentation, and pitfalls. Radiology 1990;174:297-307.

12. Harris A, Williamson TH, Shoemaker JA, Sergott RC, Spaeth GL, Katz JL. Reproducibility of color doppler imaging assessment of blood flow velocity in orbital vessels. J Glaucoma 1995;4:281-6.

13. Langham ME, Farrell RA, O'Brien V, Silver DM, Schilder P. Blood flow in the human eye. Acta Ophthalmol (Copenh) 1989;191(67 Suppl): 9-13.

14. Langham ME, Farrell RA, O'Brien V, Silver DM, Schilder P. Non-invasive measurement of pulsatile blood flow in the human eye: ocular haemodynamics. In: Lambrou GN, Greve EL, editors. Ocular blood flow in glaucoma. Amsterdam: Kugler and Ghedini Publications, 1989:93-9.

15. Silver DM, Farrell RA. Validity of pulsatile ocular blood flow measurements. Surv Ophthalmol 1994;38 (Suppl): 72-80.

16. Krakau CET. Calculation of the pulsatile ocular blood flow. Invest Ophthalmol Vis Sci 1992;33:2754-6.
17. Krakau CET. A model for pulsatile and steady ocular blood flow. Graefes Arch Clin Exp Ophthalmol 1995;233:112-8.

18. James CB, Trew DR, Clark K, Smith SE. Factors influencing the ocular pulse: axial length. Graefes Arch Clin Exp Ophthalmol 1991;229:341-4.

19. Wilke K. Effects of repeated tonometry: genuine and sham measurements. Acta Ophthalmol (Copenh) 1972; 50:574-82.

20. Trew DR, James CB, Thomas SHL, Sutton R, Smith SE. Factors influencing the ocular pulse: the heart rate. Graefes Arch Clin Exp Ophthalmol 1991;229:553-6.

21. Claridge K, Smith SE. Diurnal variation in pulsatile ocular blood flow in normal and glaucomatous eyes. Surv Ophthalmol 1994;38 (Suppl):S198-205.

22. Bill A. Blood pressure in the ciliary arteries of rabbits. Exp Eye Res 1963;2:20.

23. Alm A, Bill A. Ocular and optic nerve blood flow at normal and increased intraocular pressures in monkeys (Macaca irus): a study with radioactively labelled microspheres including flow determinations in brain and some other tissues. Exp Eye Res 1973;15:15-29.

24. Bynke HG, Schele B. On the origin of the ocular pressure pulse. Ophthalmologica 1967;153:29-36.

25. Srivastava A, Hughes LE, Woodcock JP, et al. Vascularity in cutaneous melanoma detected by Doppler sonography and histology: correlation with tumour behaviour. Br J Cancer 1989;59:89-91.

26. Bosem ME, Lusky M, Wienreb RN. Short term effects of levobunolol on ocular pulsatile flow. Am J Ophthalmol 1992;114:280-6.

27. Yang YC, Hulbert MFG, Batterbury M, Clearkin LG. Pulsatile ocular blood flow measurements in healthy eyes: reproducibility and reference values. J Glaucoma (in press).

28. Trew DR, Smith SE. Postural studies in pulsatile ocular blood flow. II. Chronic open angle glaucoma. Br J Ophthalmol 991;75:71-5.

29. James CB, Smith SE. Pulsatile ocular blood flow in patients with low tension glaucoma. Br J Ophthalmol 1991;75:466-70.

30. Langham ME, Grebe R, Hopkins S, Marcus S, Sebag M. Choroidal blood flow in diabetic retinopathy. Exp Eye Res 1991;52:167-73.

31. Hopkins SD. Ocular haemodynamics in cataractous eyes. Acta Ophthalmol (Copenh) 1989;191 (67 Suppl): 43-8.

32. Srivastava A, Hughes LE, Woodcock JP, Sheddon EJ. The significance of blood flow in cutaneous malignant melanoma demonstrated by Doppler flowmetry. Eur J Surg Oncol 1986;12:13-8.

33. Claridge KG, James CB. Ocular pulse measurements to assess pulsatile blood flow in carotid artery disease. Br J Ophthalmol 1994;78:321-3.

34. Hitchings RA. The ocular pulse. $\mathrm{Br} \mathrm{J}$ Ophthalmol 1991;75:65.

35. James CB. Effect of trabeculectomy on pulsatile ocular blood flow. Br J Ophthalmol 1994;78:818-22.

36. Yang YC, Hulbert MF. The effect of trabeculectomy on pulsatile ocular blood flow [letter]. Br J Ophthalmol 1995;79:507-8. 